\title{
Impaired mental simulation of specific past and future personal events in non-depressed multiple sclerosis patients
}

\author{
Alexandra Ernst ${ }^{\mathrm{a}}$, Frederic Blanc ${ }^{\mathrm{b}}$, Jerome de Seze ${ }^{\mathrm{b}}$, Francois Sellal ${ }^{\mathrm{c}}$, Bruno Chauvin ${ }^{\mathrm{d}}$, Liliann Manning ${ }^{\mathrm{a}}$ \\ ${ }^{a}$ Cognitive Neuropsychology and Physiopathology of Schizophrenia (INSERM U1114), University of Strasbourg, Strasbourg, France \\ ${ }^{b}$ Neurology Department, University Hospital of Strasbourg, ICube Laboratory (CNRS; UMR 7357), Strasbourg, France \\ ${ }^{c}$ University Hospital of Colmar, Colmar, France \\ ${ }^{d}$ Psychology of Cognition Laboratory (EA 4440), University of Strasbourg, Strasbourg, France
}

\begin{abstract}
The aims of the present study were (i) to explore autobiographical memory and episodic future thought in multiple sclerosis (MS), using Levine's Autobiographical Interview; (ii) to investigate the influence of the Interview's high retrieval support condition (the specific probe phase) on MS patients' past and future simulations and (iii) to obtain the patients' estimations of their own difficulties, during the test, and in everyday life. To that end, we examined 39 non-depressed relapsing-remitting MS patients and 34 healthy subjects matched for gender, age and education level. The 73 participants underwent an adapted version of the Autobiographical Interview in two conditions: remembering and imagining personal events. The group of patients also underwent an extended neuropsychological baseline, including particularly, anterograde memory and executive functions. The results showed that the MS patients' scores on the baseline were mildly or not impaired. On the contrary, the Autobiographical Interview measure, i.e., the mean number of internal details, for each of the two phases of the test - free recall and specific probe - was significantly lower in simulated past and future events in comparison with the healthy controls. Within each group, autobiographical memory performance was superior to episodic future thought performance. A strong positive correlation was observed between past and future mental simulation scores in both groups.
\end{abstract}

In conclusion, our results showed, for the first time, the co-occurrence of deficit of remembering the past and imagining the future in MS patients. They also showed more difficulty in imagining future events than remembering past events for both patients and normal controls. MS being a neurological condition very frequent in the young adult population, the clinical considerations of our study might be of interest. Indeed, they give rise to new insights on MS patients' daily life difficulties related to impaired mental simulation of personal events despite general abilities, including anterograde memory, only mildly or not impaired.

\section{Introduction}

The occurrence of cognitive impairment in multiple sclerosis (MS) has been established for the past 20 years $[10,38]$, covering a wide range of cognitive functions such as anterograde memory, executive functions, attentional processes, and information processing speed, among others [44].

More recently, the investigation of autobiographical memory (AM) in MS patients has attracted some attention. Briefly stated, AM is the capacity of reliving detailed events, within their specific spatio-temporal context, as they are remembered [58]. An overview of AM studies in MS [20,21,25,35,37] shows some conflicting results, which seem to rely mainly on some methodological discrepancies, particularly, the sensitivity of the test used to assess AM in MS. The call for a stringent AM test is also motivated by the fact that notwithstanding the variability of AM test performance, MS patients' complaints are frequent. These clinical observations together with neuroanatomical and conceptual considerations (see below), led to the suggestion that AM impairment in MS patients is likely caused by a deficit of retrieval strategies rather than a loss of personal recollections [20,21]. The deficit would be then attributed to dysfunctional prefrontal regions ([7,8,30]).

From a neuroanatomical standpoint, MS patients' difficulties in remembering past events are coherent with both 
the multifocal nature of MS lesions and the widespread cerebral network recruited during AM tasks $[53,57]$. This cerebral network encompasses predominantly the left and medial cerebral regions, namely, the prefrontal cortex, medial and lateral temporal cortices, parieto-occipital regions, temporoparietal junctions and cerebellum. Consistent with these findings, the preliminary fMRI results obtained by Ernst et al. [21] showed enhanced bilateral prefrontal region activations during the initial retrieval phase of past events, which seemed to corroborate the retrieval-deficit hypothesis. Moreover, the enhanced prefrontal activation having been observed bilaterally, the suggestion was one of a compensation mechanism attempt, although insufficient to result in normal AM performance [21].

Recent neuroimaging and clinical studies have examined the ability to mentally simulate hypothetical scenarios from not only past recollections but also those that may potentially occur in the future, i.e., the relationships between remembering the past and imagining the future. Imagining future personal events or episodic future thought (EFT; [55]; see [45] for a review), is the ability to mentally pre-experience a personal event that may happen in the future. The relation between AM and EFT, sometimes referred to as "mental time travel" [50,51], has been extensively studied in the healthy subject (e.g. [1,8,54]), revealing a common cerebral network assumed to reflect the engagement of similar cognitive processes. Indeed, AM and EFT involve not only the selfreferential processes, but also the retrieval and binding of details into a coherent event representation, which include conceptual and sensory information, with a strong reliance on visual imagery processes [1].

Subsequently, in clinical studies, it was surmised that EFT would also be deficient whenever AM impairment had been observed. Investigations carried out in several clinical conditions such as Alzheimer disease (e.g. [3]), amnesic mild cognitive impairment (e.g. [22]), schizophrenia (e.g. [15]), depression (e.g. [62]), or medial temporal lobe amnesia (e.g. [24,39]) confirmed the co-occurrence of deficit in the simulation of past and future personal events.

To our knowledge, EFT has never before been explored in MS patients despite the characteristic onset of the illness occurring during young adulthood and despite the clinical value of the central role of mental time travel in everyday life. Indeed, AM contributes to the construction and the continuity of the personal identity, plays at least two social roles, the development of new relationships and the nurturing of existing ones, and involves also a directive function, where the past serves as a basis for guiding present and future behaviours [9,40], With regard to EFT, three main functional benefits have also been described [55] comprising a role in (i) coping skills for stressful events, with an implication in emotion regulation and problem solving; (ii) goal achievement, for which the act of imagining various scenarios helps to reach the desired goal; and finally, (iii) the implementation of intentions, for which a higher likelihood of completing an action is observed when there is an intention to implement this action. Furthermore, D'Argembeau, Lardi \& Van der Linden [17] suggested that EFT, similarly to AM and, particularly, self-defining EFTs contribute to a person's sense of self and, together with self defining AMs, give rise to a strong sense of personal continuity over time.

Based on both the abovementioned works on the interdependence between AM and EFT in terms of common cognitive processes, and previous reports on AM in MS patients, the aims of the present study were (i) to explore autobiographical memory and episodic future thought in non-depressed relapsing-remitting MS patients, (ii) to investigate the influence of a high retrieval support condition on MS patients' past and future simulations, and (iii) to obtain the patients' reports about their difficulties during the test, and in everyday life. To that end, we probed the ability of MS patients to generate future episodic events, as well as to remember recent past events and remote past events from across lifespan. The rationale for exploring remote past events relied on the importance of having a comprehensive view of MS patients' AM performance. Effectively, taking into account the suggestion of an AM retrieval-deficit hypothesis in MS patients, exploring only recent past events, which are generally more accessible memories, could result in an underestimation of the AM impairment. A similar pattern of impairment was expected between future events and both time intervals, and recent and remote past events in MS patients. Complementarily, a correlation between AM and EFT scores in our patients was also expected.

As the AM test sensitivity is a main concern in the exploration of AM functioning, we carried out the research with the Autobiographical Interview (AI; kindly communicated by Brian Levine to one of us, LM), based on its sensitivity to detect AM and EFT deficits in different clinical populations (e.g. [3,19,22]). Moreover, as the AI includes both a free recall and a high retrieval support condition (i.e. the specific probe phase), a further aim was to investigate the influence of this last condition on AM and EFT performance in MS patients. Finally, we also aimed at obtaining the patients' self-reports on their difficulties during the mental simulation testing, and also more generally in their everyday life. 


\section{Methods}

\subsection{Participants}

Thirty-nine patients with definite MS according to the McDonald criteria [31 ] were recruited at the Neurology Units of two French hospitals (Strasbourg and Colmar). Inclusion criteria were as follows: RR-MS disease course, Expanded Disability Status Scale (EDSS; [27]) score $\leq 5$, absence of major signs of depression according to the Montgomery and Asberg Depression Rating Scale (MADRS; [33]: significant clinical threshold score $\geq$ $15)$, and no recent exacerbation of MS symptoms.

Thirty-four healthy controls matched for gender, age and education level were also recruited. Exclusion criteria for all the participants included documented psychiatric illness, neurological disorder (other than MS for the patient group) and poor knowledge of French. Demographic and clinical data are summarised in Table 1. The present study was approved by the 'Committee for Protection of Persons' (CPP/CNRS No. 07023) and we complied with the APA ethical standards.

\subsection{Neuropsychological baseline examination}

The patients completed a comprehensive baseline examination in order to verify their cognitive status allowing us to control any potential confounding variables on the AM and EFT performance. It comprised the following functions, general verbal abilities, tested with the Verbal IQ, short form [4] of the WAIS-111 [61], and nonverbal reasoning assessed using the Advanced Progressive Matrices Set 1 [41]. Anterograde memory was examined with the Rey Auditory Verbal Learning Test (RAVLT; [42]), and the Rey-Osterrieth Complex Figure (ROCF; $[36,43])$. The executive functions were probed by means of the National Hospital, London's phonological and categorical fluency tests, the Brixton Spatial Anticipation Test [11], the Tower of London Test [46,48], and the Cognitive Estimation Task [47]. The attentional abilities and information processing were assessed using the Information Processing Speed Test from the Adult Memory Information Processing Battery (AM1PB; [13]), the Stroop Test [49], and the Months Backwards Test (National Hospital, London). Language was tested with the Deno 100 test [26], and the visuo-perceptual and visuo-spatial abilities with the Silhouettes and Cube Analysis Sub-Tests from the Visual Object and Space Perception Battery (VOSP; [60]). Additionally, the effects of fatigue in everyday life were assessed using the "Echelle de Mesure de l'Impact de la Fatigue", (EM1F-SEP; [18]).

Finally, in the interest of obtaining qualitative information about MS patients' perception of potential AM/EFT difficulties during the assessment, and also in everyday life, we proposed a semi-structured interview (described in [20]) at the end of the AI session.

\subsection{Assessment of mental simulation of past and future events}

The assessment of AM and EFT was based on the AI described in Levine et al. [28]. The AI general instructions for both AM and EFT conditions were as follows: to retrieve/imagine personal unique events, temporally and contextually specific, occurring over minutes to hours and to generate freely as many details as possible about the event. We used the adapted version of the AI [2,3], introducing cue-words for

AM (e.g. car, restaurant) and EFT (e.g. family, park), which allowed us to carry out comparable examinations for both temporal directions. Additionally, based on previous studies [5,21,29], we modified the number of events to be retrieved from one (in the standard version) to three past events per life period explored [i.e. four or five life periods, depending on the subject's age; 0-11 years, 12-20 years, 21 to (current age -1) or 21-35 years, 36 to (current age -1) and the previous year]. The aim of testing a greater number of past recollections was to assess more comprehensively the retrieval process and to avoid the likelihood of the patients providing only the most accessible detailed recollections [5,29]. With regard to the EFT testing, following Addis et al. [3], we instructed the participants to generate five events, which could plausibly occur during the coming weeks or months or any interval during the next year. These instructions attempted to minimise any potential conscious avoidance of EFT in the patients due to the uncertainty surrounding disease progression.

The order of presentation between AM and EFT tasks was counterbalanced as were the order of the cue-words and the past life periods. To encourage mental simulation of past and future personal events, participants were informed that the cue-words were intended to be used flexibly (e.g. the cue-word "train" could result in a personal recollection of a journey by any other means of transport). No time limit was set to avoid the potential influence of decreased cognitive processing speed on AM/EFT performance for MS patients. 
General probes (e.g. 'is there anything else you can tell me?') were used to clarify instructions if necessary and to encourage evocation of supplementary details. It was followed by the specific probe phase, which is a structured interview to elicit additional details that were not spontaneously recalled. Specific probe questions are organised into different categories (time: e.g. year, month; place: building; sensory information: sounds, event duration; emotion: feeling).

The AI session was audio-recorded for later transcription and scoring following the standardised Levine et al. [28] procedure. Two phases of recall were analysed, the free recall and the specific probe phases. However, for the subsequent statistical analyses, the specific probe phase scores were analysed cumulatively with the free recall phase scores.

After the identification of the central event, details were classified as internal details (i.e., an episodic detail related to the central event) or external (i.e., non-episodic information such as semantic details, metacognitive statements, repetitions or else episodic details unrelated to the central event). For each participant, the number of internal details was averaged across the 12 or 15 past events for the AM, or the three past events for the previous year, and across the five future events for the EFT conditions for each recall phase. Since Ernst et al. [20] highlighted an impairment of the episodic component of AM, whereas the personal semantics was preserved, only the mean number of internal details was considered for the following statistical analyses.

The interrater reliability was verified for $10 \%$ of the past and future events, which were scored by a second scorer, following Levine et al.'s [28] procedure. Coefficients for all measures showed high interrater reliability (between 0.82 and 0.99).

\subsection{Statistical analyses}

Mean scores obtained by the MS patients for each neuropsychological baseline test were calculated.

Differences between the MS patients and healthy controls in AM/EFT were analysed for the mean number of internal details by running mixed ANOVAs to explore main effects of group (MS vs. healthy controls), temporal direction (past vs. future) and recall phase (free vs. specific probe phases), as well as relevant interactions. This analysis was conducted for the remote past events for the AM condition (i.e. all the life periods, with the exclusion of the previous year) vs. the EFT condition. Then, a similar complementary analysis including only the previous year for the AM condition vs. the EFT condition was also conducted by means of a mixed ANOVA to obtain a comparison of the performance (mean number of internal details) with a matched temporal interval.

Pearson coefficient correlations between AM (remote and recent memories) and EFT performance within the two groups were calculated for the mean number of internal details across the two phases of recall.

Table 1 Demographic and clinical data: Mean and (standard deviation) for the MS patient and the healthy control groups.

\begin{tabular}{lccc}
\hline & MS patients & Healthy controls & Statistical analysis \\
\hline $\mathrm{N}$ & 39 & 34 & - \\
Age in years & $39.77($ SD 7.88$)$ & $37.85($ SD 11.64$)$ & $\mathrm{t}=0.83 ; \mathrm{p}=0.41$ \\
Education in years & $13.38(\mathrm{SD} 2.15)$ & $14.21(\mathrm{SD} 2.71)$ & $\mathrm{t}=-1.44 ; \mathrm{p}=0.15$ \\
Sex (ratio female/male) & $33 / 6$ & $26 / 8$ & $\chi^{2}=0.77 ; \mathrm{p}=0.37$ \\
EDSS & $2.54(1.38)$ & n.a. & - \\
[range] & {$[0-5]$} & & - \\
Duration of MS in years & $12.07(7.56)$ & n.a. & - \\
Number of DMD treatment & $1.0($ SD 0.0$)$ & n.a. & - \\
\hline
\end{tabular}

EDSS: the Expanded Disability Status Scale; SD: standard deviation; and n.a.: not applicable

\section{Results}

The patients' neuropsychological baseline scores were in the normal range (i.e. threshold: either z-score -1.65 or the 5 th percentile, depending on the normative data of each test, of which the source is indicated for each test below Table 2) for all cognitive functions, with two exceptions, planning abilities and cognitive estimations (see 
Table 2).

\subsection{Remote past events vs. future events}

Regarding the mean number of internal details (Fig. 1), mixed ANOVA revealed a main effect of group $F(1$, $142)=42.07, p<0.001, \eta_{P}^{2}=0.23$, which reflected that, irrespective of the temporal direction or the recall phase, MS patients' scores were significantly lower than those of the healthy controls. A main effect of the temporal direction was also highlighted $F(1,142)=16.15, \mathrm{p}<0.001, \eta^{2} \mathrm{P}=0.11$, showing that the mean number of internal details provided in the AM condition was higher than for the EFT condition, whatever the group or the recall phase. No significant Group x Temporal direction interaction was evident $(\mathrm{p}=0.66)$.

The main effect of the recall phase was also observed, $F(1,142)=897.74, p<0.001, \eta^{2}{ }_{\mathrm{P}}=0.86$, with a greater number of internal details provided during the specific probe than during the free recall phase. The specific probe phase enabled a greater increase of the mean number of internal details for the healthy controls (vs. the MS patients; $\mathrm{p}<0.001$ ), irrespective of the temporal direction, as revealed by the significant Recall Phase $\mathrm{x}$ Group interaction, $F(1,142)=6.31, \mathrm{p}=0.01, \eta^{2} \mathrm{P}=0.04$. Moreover, a differential effect of the specific probe phase on AM and EFT was displayed, with a significant Recall Phase $\mathrm{x}$ Temporal direction interaction $F(1,142)=4.69$, p $=0.03, \eta^{2} \mathrm{P}=0.03$, showing a lower increase of the mean number of internal details during the specific probe phase for the EFT condition ( $\mathrm{p}<0.001$ ). No significant 3 -way interaction was obtained.

Table 2 Neuropsychological baseline examination scores: Mean (and SD) for the MS patient group in comparison with the normative data of each test (mean and SD score, 5th percentile or cut-off depending on the test).

\begin{tabular}{|c|c|c|}
\hline & MS group scores Mean (SD) & Normative data \\
\hline Verbal IQ & $98.46(12.73)$ & $100.00(15.00)$ \\
\hline PM12 & $9.23(1.74)$ & 5 th percentile $=3$ \\
\hline \multicolumn{3}{|l|}{ RAVLT } \\
\hline -Total mean number of words & $12.36(1.34)$ & $10.58(2.16)$ \\
\hline -Delayed recall & $13.74(1.72)$ & $11.1(2.8)$ \\
\hline \multicolumn{3}{|l|}{ ROCF } \\
\hline -Copy & $35.67(0.72)$ & $32.83(3.10)$ \\
\hline -Immediate recall & $24.73(4.60)$ & $20.3(7.42)$ \\
\hline -Delayed recall & $24.71(4.62)$ & $19.28(7.29)$ \\
\hline Déno 100 & $97.53(3.51)$ & $>85 / 100$ \\
\hline \multicolumn{3}{|l|}{ Stroop } \\
\hline -Colours (score $\mathrm{T}$ ) & $46.0(8.03)$ & $50.00(10.00)$ \\
\hline -Words (score T) & $44.64(7.56)$ & $50.00(10.00)$ \\
\hline -Interference (score $\mathrm{T})$ & $47.71(10.55)$ & $50.00(10.00)$ \\
\hline -Interference score (score $\mathrm{T})$ & $51.71(7.82)$ & $50.00(10.00)$ \\
\hline Months Back (sec) & $10.79(3.35)$ & $<15$ \\
\hline \multicolumn{3}{|l|}{ Tower of London } \\
\hline -Score & $8.15(1.69)^{* *}$ & $>9$ \\
\hline -Time index & $18.10(3.60)$ & $<25$ \\
\hline Brixton (number of errors) & $14.74(6.19)$ & $<25$ \\
\hline Cognitive Estimation Task & $4.28(3.20)^{* *}$ & $<4$ \\
\hline \multicolumn{3}{|l|}{ Verbal Fluency } \\
\hline -Categorical & $20.07(4.81)$ & $>15$ \\
\hline -Phonological & $13.18(3.22)$ & $>9$ \\
\hline \multicolumn{3}{|c|}{ Information Processing Speed (AMIPB) } \\
\hline -Cognitive & $55.72(13.07)$ & $63.6(15.01)$ \\
\hline -Motor & $48.77(9.35)$ & $49.3(12.7)$ \\
\hline -Error percentage & $2.80(3.16)$ & $3.1(3.4)$ \\
\hline -Corrected score & $62.43(15.45)$ & $72.2(18.4)$ \\
\hline \multicolumn{3}{|l|}{ VOSP } \\
\hline -Silhouettes & $23.51(3.65)$ & $>16$ \\
\hline -Cube Analysis & $9.79(0.52)$ & $>6$ \\
\hline MADRS & $6.05(4.10)$ & $<15$ \\
\hline EMIF-SEP (total) & $48.35(14.94)$ & $57.7(22.1)$ \\
\hline
\end{tabular}


Verbal IQ[61]; PM12: Progressive Matrices 12 (National Hospital, London); RAVLT: Rey Auditory Verbal Learning Test (Compendium of Neuropsychological Tests, 3rd Ed., 2006); ROCF: Rey-Osterrieth Complex Figure; Deno 100 [26]; Stroop Test [23]; Months Back (National Hospital, London); Tower of London [12]; Brixton [11]; Cognitive Estimation Task [47]; Verbal Fluency (National Hospital, London); AMPB: Information Processing Speed Test from the Adult Memory Information Processing Battery [13]; VOSP: Visual Object and Space Perception [60]; MADRS: Montgomery and Asberg Depression Rating Scale [33]; EMIF-SEP: Echelle de Mesure de l'Impact de la Fatigue [18]; and **scores under the normal range.

Fig. 1. Mean number of internal details produced by the MS patients and the healthy controls for the remote past events of the AM condition and the EFT conditions during the free recall $(F R)$ and the specific probe $(S P)$ phases.

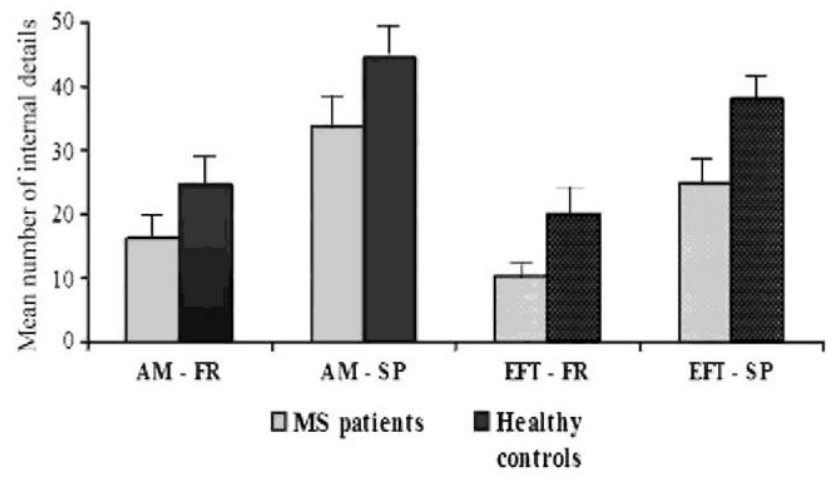

Fig. 2. Comparison of the mean number of internal details produced by the MS patients and the healthy controls for the recent past events of the AM condition and the next year (EFT condition) during the free recall (FR) and the specific probe $(S P)$ phases.

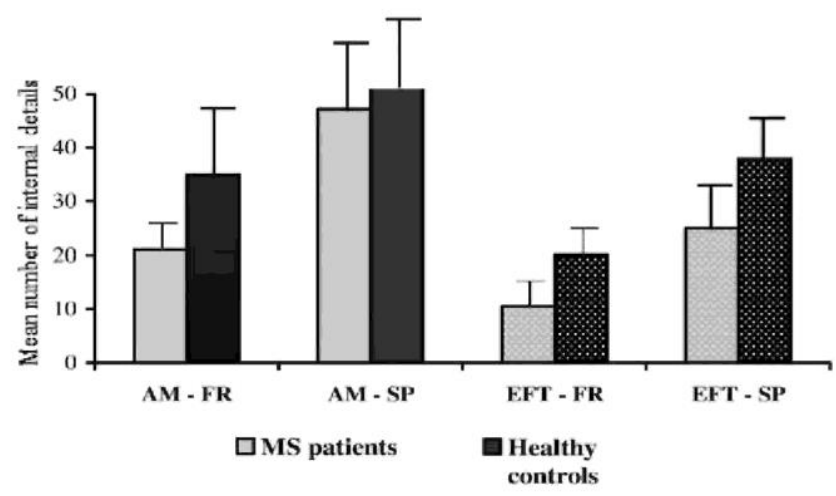

\subsection{Recent past events vs. future events}

Considering only the previous year (AM condition) and the EFT condition, analyses for the mean number of internal details showed the main effect of group, $F(1,142)=29.62, \mathrm{p}<0.001, \eta^{2}{ }_{\mathrm{P}}=0.17$, with lower scores for the MS patients in both conditions (Fig. 2). A main effect of temporal direction was replicated, with AM scores superior than EFT scores, $F(1,142)=65.99, \mathrm{p}<0.001, \eta^{2} \mathrm{p}=0.32$, in both group. No significant Group $\mathrm{x}$ Temporal direction was observed $(\mathrm{p}=0.65)$.

Irrespective of the group and the temporal direction, the mean number of internal details provided by the subjects after the specific probe phase was significantly higher than those of the free recall phase, as evidenced by the main effect of the recall phase, $F(1,142)=773.54, \mathrm{p}<0.001, \eta_{\mathrm{P}}^{2}=0.84$. A significant Recall Phase $\mathrm{x}$ Temporal direction has been shown, $F(1,142)=46.49, \mathrm{p}<0.001, \eta_{\mathrm{P}}{ }_{\mathrm{P}}=0.24$, with a greater benefit of the specific probe phase on past recall than on future simulation $(\mathrm{p}<0.001)$. No other interaction effect was significant. 


\subsection{Correlations between AM and EFT performance}

Finally, the correlations between the AM and EFT scores within each group were carried out. For the MS patients, the mean number of internal details provided for the remote past events of the AM condition correlated positively with the EFT results during the free recall $(\mathrm{r}=0.49, \mathrm{p}=0.002)$ and the specific probe phases $(\mathrm{r}=0.58$, $\mathrm{p}<0.001)$. Comparable results were observed when the correlation analyses were run between the recent past events and the next year for the mean number of internal details $(r=0.54, p<0.001)$ during the free recall phase, as well as during the specific probe phase (mean number of internal details: $r=0.41, p=0.01$ ).

The same pattern of results was obtained for the healthy controls for the free recall (mean number of internal details: $r=0.74, p<0.001$ ) and the specific probe phases (mean number of internal details: $r=0.70, p<0.001$ ) when taking into account the remote past events and the future events. Regarding the relationship between EFT and the recent past events of the AM condition performance, strong correlations were obtained during both the free recall phase for the mean number of internal details $(\mathrm{r}=0.75, \mathrm{p}<0.001)$ and the specific probe phase $(\mathrm{r}=$ $0.75, \mathrm{p}<0.001)$

\subsection{Qualitative self-assessment of AM and EFT performance}

With regard to the semi-structured interview, the qualitative results gathered in the form of patients' comments (see Appendix A for extracts from patients' interviews), corroborated the aforementioned quantitative results. Generally, MS patients verbalised difficulties for the retrieval/imagination of a specific event, and also an impoverishment of details, conducting to low vividness and emotional intensity throughout the evocation of an event during the testing session, and also in some real life situations. Additionally, most of our patients' comments also stated that the EFT task was experienced as being harder than the AM task.

\section{Discussion}

We have documented the mental simulation of past and future personal events in non-depressed RR-MS patients. The results showed a mildly impaired neuropsychological baseline performance together with significantly poorer scores, compared with controls, in the mental simulation of past and future personal events. These findings are in accord with our previous work [20] on AM in MS, using the Crovitz Test in a relatively small sample. The present results support the notion that AM and EFT, in the light of the relatively well preserved neuropsychological baseline, could be particularly sensitive to MS neuropathology. Our results might modify the often absent assessment of AM and EFT in clinical routine, since they suggest new insights into how MS patients' everyday life is affected by impaired mental time travel capacity (see below).

Turning to the AM and EFT performance, compared with healthy subjects, the patients generated significantly fewer episodic details in both remembering past events and imagining future episodes, with a comparable degree of impairment for both temporal directions. Moreover, for both remote and recent past events, our group of MS patients showed lower scores than healthy controls, replicating our previous findings of AM impairment in MS patients covering all the life periods [20]. Additionally, in every case, taking into account remote or recent past events, lower scores were observed for the simulation of future events.

This AM/EFT deficit was observed for the two retrieval conditions (i.e. free recall and specific probe phases). Although a high retrieval support with specific cues led the patient group to a relative performance improvement, it turned out to be insufficient since the scores remained under the healthy controls' results. In other words, it seemed that the healthy controls benefited to a greater extend of the specific cueing than the MS patients, irrespective of the temporal direction of the mental simulation. However, the greater benefit of the specific cueing for healthy controls was observed only when all the AM life periods were taken into account (it disappeared in the one year past/future condition). This finding suggests that a high retrieval support could be particularly effective for MS patients for the more recent past events, which are also, in general, more easily detailed [14]. Differential results between the two recall phases on AM scores, showing an improvement after the specific cueing, have been described in different clinical populations, especially patients presenting with prefrontal cortex lesions, in a context of retrieval strategy impairment, relying mostly on the prefrontal regions $[28,32,52]$. In contrast, in the context of amnesic patients presenting with medial temporal lobe or diencephalic damage, the effect from a higher retrieval support is inefficient or modest, at best [28,34].

The smaller number of details produced during the EFT condition compared with the AM condition was observed in both groups. This result is in accord with previous investigations, showing that past events are more detailed than future events in healthy subjects [2,14], and also in patients presenting with an AM/EFT 
impairment (e.g. [3]). Addis and her colleagues interpreted their findings in terms of a more important cognitive demand in pre-experiencing an event compared with remembering an event. The increased cognitive demand would tax above all executive functions because EFT requires the extraction and recombination of an infinite assortment of details into a novel event, whereas AM consists in the recapitulation of a more limited pool of details [6]. This notion is in accord with De Vito et al.'s [19] study, showing impaired EFT performance with preserved AM scores in Parkinson disease patients, who present with a characteristic dysexecutive syndrome. De Vito et al.'s aim was to assess EFT performance in patients, who, besides their dysexecutive condition, were nonamnesic and therefore, different from our autobiographical amnesic MS patients. Despite this difference both studies have documented impaired EFT, which suggests the particularly vulnerable quality of the ability to simulate personal future events.

The effect of specific cueing on the simulation of future events was lower compared with cueing either recent or remote past events, in both groups. This finding could reflect the fact that the specific cueing is more effective when the number of possibilities is limited. For example, when imagining a future dinner with friends, the issue about the menu would give rise to countless possibilities. On the contrary, when remembering a dinner with friends, virtually any remembered detail as a response to the specific probe (e.g. X tasted for the first time sushi; we were with $\mathrm{N}$, who loves pasta) may restrict the possibilities of the menu.

Taken together, the two mentioned findings, i.e., the benefit of a high retrieval support during the AI specific probe phase on the mental simulation tasks, and the poorer performance for EFT relative to AM condition could be seen as an additional argument for the retrieval deficit hypothesis in MS patients.

Despite the results being coherent with the non-MS literature (e.g., [59], the investigation of EFT in MS patients raises an important issue owing to the clinical presentation of the disease, and especially for RR-MS patients. Namely, because of the uncertainty surrounding MS evolution, one could argue that the EFT deficit, found in the present study, could reflect psychological factors such as a conscious decision of the patient not to engage in future projects despite the absence of depressive symptoms. However, the positive correlation observed between AM and EFT deficit in the patient group suggests that the EFT performance reflected a cognitive deficit. We are aware that a psychological influence cannot be ruled out, but practically all the patients reported cognitive difficulties, particularly so in relation to the EFT. We observed no examples of psychological inhibition or conscious avoidance of EFT during the semi-structured interview, which was probably due to the relatively short interval in which the patients were asked to mentally simulate potential scenarios (see Appendix A).

Bearing in mind the essential role of AM and EFT in everyday life [16,40,55], the present findings suggest some clinical issues. In the first place, besides our MS patients' occasional complaints, the semi-structured interview showed that they were well (and accurately) aware of their difficulties and able to verbally articulate them to convey a clear message. Given the fact that the quantitative results endorsed the qualitative assessment, and that the patients alluded to real life not infrequently, it could be useful to introduce the same type of questions at the end of the AI and similar tests, in routine clinical practise. Moreover, in previous studies [20,21], a cognitive facilitation programme has been proposed to improve AM in MS patients, which resulted in normalised AM performance, and whose benefits were efficiently transferred to daily-life functioning. Whether those positive AM results would be also observed in the EFT mental simulation is again an issue of clinical interest. Indeed, bearing in mind the role of EFT in coping for stressful events and goal achievement, improving the EFT ability could be particularly useful vis-a-vis some particular requirements in the course of the disease.

To conclude, the present findings showed that RR-MS patients had cognitive difficulties in the mental simulation of past and future personal events similar to other clinical conditions, and a cognitive profile suggesting that in MS, these difficulties can be more taxed than several other cognitive functions that were also explored. They showed too that qualitative evaluation of AM/EFT difficulties has clinical value.

Our results encourage further investigation, namely, trying to understand how simulated future events that are known to be important in real life are modulated by the course of the disease. Any research along these lines would be closely related to current investigations centred in understanding why simulated future events are important in real life [56]. On a more matter-of-fact level, this study also encourages the replication of the results with a larger sample of MS patients and the introduction of functional neuroimaging data. In relation to the latter, one remaining question concerns the possibility of a compensation mechanism attempt during an EFT task (although insufficient to result in normal performance), as previously observed for AM performance in MS patients [21]. If that were the case, a potentially interesting investigation would be to explore the similarities and differences between AM and EFT in the setting up of cerebral compensation mechanisms. 


\section{Conflict of interest}

The authors report no conflict of interest.

\section{Acknowledgements}

We are grateful to the "Fondation pour la Recherche sur la Sclerose en Plaques" (ARSEP; Ile de France; grant accorded to L.M.) for research funding, and to the Ministry of National Education and Research (A.E.'s PhD grant).

We thank Catherine Vinet-Gasse for her contribution in testing patients, Anne Botzung for her contribution in testing healthy controls and Nathalie Heider, Sabine Graves, Florine Ernwein, Alexandra Clerc-Renault, Emilie Montaut and the 18 Masters students for their contribution in transcribing the AI audio-recordings.

\section{Appendix A}

Excerpts of patients' comments during the semi-structured interview presented at the end of the Autobiographical Interview.

\section{Autobiographical memory}

IB: I'm forever hesitating when I recollect, I always hesitates. That's very..., it's horrible, because I have lots of memories somewhere, but they are snippets of memories (...). The only thing that could, eventually, help me is to talk with the people who were there... also to reconstruct myself, to remember or to go back to people, whose ... to things that I have already forgotten, actually. It doesn't always come back and that, that is very upsetting. I have this feeling that my memories disappear and it pains me.

MFW: It's difficult because it's terrible not be able to remember... if someone tells you anything, I don't know, asks you "where were you?" that kind of questions... I mean, sometimes they can keep asking you endlessly. It's the same with things that I should absolutely know, but I don't. It's very hard sometimes because deep inside I tell myself "you look like an idiot and yet you know that stuff, but I know no longer, not easy really, it's hard.

NK: I'd say that for some memories, I've given you quite a lot of details, but for other memories I gave you a general idea, one important thing, and I just couldn't get the details as I should have, of course I should have (...) There are many things that I can't any longer remember (...) What really gets on my nerves is to have these flashes and I can't say when it was, who it was...

VW: I can't get it, can't get back to the film. No, no, no. Sometimes there are some things that... the event is ... as if I had erased it, as if] had never been there, as if... My friend remembers "Come on! There was this and that, we went to..." And I say "Really? But I was not there". "Yes you were" And I really feel like I have nothing to do with the event.

\section{Episodic future thought}

NG: Not OK, also because I have some difficulties to project myself into the future, difficult to imagine a scenario that could happen... it's always quite complex because when dealing with quite specific things you never know how people will react in that situation. That's why it's always a little bit blurred.

CL: I'd say that, well it's not clear for me because I've really tried to get it, really tried to find the ... no, there were no details. Let's say that I've just taken bits of what I've lived at some moments and put them in the project.

VF: It's no longer flashes or images I have; I think it's something in between. It's more static, if there is a little bit of movement it doesn't last long (...). Yes, I was saying things that have already happened; I know I had to dissociate them...

IN: I had to look for longer, anyway, to think more...There is this stuff that comes more or less spontaneously, but for other things I really need to look for...

CE: Imagine myself in a scenario, seeing all that surrounds me is not easy compared to an experienced situation. To find the moments, let's say, it's not that hard, but after that, to describe all the things that go with it, people, 
context of the room, the place...

IJ: It's somewhat like the past, it remains blurred.

MFW: It's clear that I'm unable to project myself...I just can't, well, it's near future, if I try to think... I'll only be able to tell you one... but no, no details for me. No, no details, well I'd say it's less than a flash, it's an act, if you know what I mean (...) But otherwise, it's for sure I can't project myself, I just can't.

\section{References}

[1] Addis DR Wong AT, Schacter DL Remembering the past and imagining the future: common and distinct neural substrates during event construction and elaboration. Neuropsychologia 2007;45:1363-77.

[2] Addis DR, Wong AT, Schacter DL. Age-related changes in the episodic simulation of future events. Psychol Sci 2008;19:33-41.

[3] Addis DR, Sacchetti DC, Ally BA, Budson AE, Schacter DL. Episodic simulation of future events is impaired in mild Alzheimer's disease. Neuropsychologia 2009;47:2660-71.

[4] Axelrod BN, Ryan JJ, Ward LC. Evaluation of seven-subtest short forms of the Wechsler Adult Intelligence Scale - III in a referred sample. Arch Clin Neuropsychol 2011;16:1-8.

[5] Barnabe A, Whitehead V, Pilon R Arsenault-Lapierre G, Chertkow H. Autobiographical memory in mild cognitive impairment and Alzheimer's disease: a comparison between the Levine and Kopelman interview methodologies. Hippocampus 2012;22:1809-25.

[6] Berntsen D, Bohn A Remembering and forecasting: the relation between autobiographical memory and episodic future thinking. Mem Cogn 2010;38:265-78.

[7] Botzung A, Denkova E, Ciuciu P, Scheiber C, Manning L The neural bases of the constructive nature of autobiographical memories studied with a self-paced fMRI design. Memory 2008;16:351-63.

[8] Botzung A Denkova E, Manning L Experiencing past and future personal events: functional neuroimaging evidence on the neural bases of mental time travel. Brain Cogn 2008;66:202-12.

[9] Bluck S. Autobiographical memory: exploring its functions in everyday life. Memory 2003;11:113-23.

[10] Brassington JC, Marsh NV. Neuropsychological aspects of multiple sclerosis. Neuropsychol Rev 1998;8:43-77.

[11] Burgess P, Shallice T. The Hayling and Brixton tests. Test manual. Bury St Edmunds, UK: Thames Valley Test Company; 1997.

[12] Collette F, Van der Linden M. Une adaptation du test de la tour de londres. Bruxelles: Service de Neuropsychologie, Universite de Liege; 1993.

[13] Coughlan A Hollows S. The Adult Memory and Information Processing Battery. Leeds: Saint James Hospital; 1985.

[14] D'Argembeau A, van der Linden M. Phenomenal characteristics associated with projecting oneself back into the past and forward into the future: influence of valence and temporal distance. Conscious Cogn 2004;13:844-58

[15] D'Argembeau A, Raffard S, Van Der Linden M. Remembering the past and imagining the future in schizophrenia. J Abnorm Psychol 2008;117:247-51.

[16] D'Argembeau A, Renaud O, Van der Linden M. Frequency, characteristics and functions of future-oriented thoughts in daily life applied cognitive psychology. Appl Cogn Psychol 2011;25:96-103.

[17] D'Argembeau A Lardi C, Van der Linden M. Self-defining future projections: exploring the identity function of thinking about the future. Memory 2012;20:110-20.

[18] Debouverie M, Pittion-Vouyovitch S, Louis S, Guillemin F. Validity of a French version of the fatigue impact scale in multiple sclerosis. Mult Scler 2007;13:1026-32.

[19] De Vito S, Gamboz N, Brandimonte MA, Barone P, Amboni M, Delia Salla S. Future thinking in Parkinson's disease: an executive function? Neuropsychologia 2012;50:1494-501.

[20] Ernst A, Blanc F, Voltzenlogel V, De Seze J, Chauvin B, Manning L Autobiographical memory in multiple sclerosis patients assessment and cognitive facilitation. Neuropsychol Rehabil 2013;23:161-81.

[21 ] Ernst A, Botzung A Gounot D, Sellal F, Blanc F, De Seze J, et al. Induced brain plasticity after a facilitation programme for autobiographical memory in multiple sclerosis: a preliminary study. Mult Scler Int 2012. http://dx.doi.org/10.1155/2012/820240. 
Published in: Journal of the Neurological Sciences (2014), vol. 345, pp. 68-74.

Status: Postprint (Author's version)

[22] Gamboz N, de Vito S, Brandimonte MA Pappalardo S, Galeone F, Iavarone A, et al. Episodic future thinking in amnesic mild cognitive impairment. Neuropsychologia 2010;48:2091-7.

[23] Golden CJ. Diagnosis and rehabilitation in clinical neuropsychology. Springfield, Illinois: Charles C.Thomas; 1978.

[24] Hassabis D, Kumaran D, Vann SD, Maguire EA Patients with hippocampal amnesia cannot imagine new experiences. Proc Natl Acad Sci 2007; 104:1726-31.

[25] Kenealy P, Beaumont G, Lin tern T, Murrell RC. Autobiographical memory in advanced multiple sclerosis: assessment of episodic and personal semantic memory across three time spans. J Neuropsychol Int Soc 2002;8:855-60.

[26] Kremin H. L'acces au lexique en denomination d'images: problemes actuels. In: Manning L, editor. Neuropsychologie cognitive clinique, 47., Revue Francaise de psychologie; 2002. p. 77-91.

[27] Kurtzke JF. Rating neurological impairment in multiple sclerosis: an Expanded Disability Status Scale (EDSS). Neurology $1983 ; 33: 1444-52$

[28] Levine B, Svoboda E, Hay JF, Winocur G, Moscovitch M. Aging and autobiographical memory: dissociating episodic from semantic retrieval. Psychol Aging 2002;17:677-89.

[29] Leyhe T, Muller S, Milian M, Eschweiler GW, Saur R Impairment of episodic and semantic autobiographical memory in patients with mild cognitive impairment and early Alzheimer's disease. Neuropsychologia 2009;47:2464-9.

[30] Manenti R Cotelli M, Calabria M, Maioli C, Miniussi C. The role of the dorsolateral prefrontal cortex in retrieval from long term memory depends on strategies: a repetitive transcranial magnetic stimulation study. Neuroscience 2010;166:501-7.

[31] McDonald WI, Compston A Edan G, Goodl<in D, Hartung HP, Lublin FD, et al. Recommended diagnostic criteria for multiple sclerosis: guidelines from the International Panel on the diagnosis of multiple sclerosis. Ann Neurol 2001 ;50:121-7.

[32] McKinnon MC, Black SE, Miller B, Moscovitch M, Levine B. Autobiographical memory in semantic dementia: implications for theories of limbic-neocorrical interaction in remote memory. Neuropsychologia 2006;44:2421-9.

[33] Montgomery SA, Asberg M. A new depression scale designed to be sensitive to change. Br J Psychiatry 1979;134:382-9.

[34] Moscovitch M, Yaschyshyn T, Ziegler M, Nadel L. Remote episodic memory and retrograde amnesia: was Endel Tulving right all along? In: Tulving E, editor. Memory, consciousness, and the brain: the Tallinn Conference. New York, NY, US: Psychology Press; 2000. p. $331-45$.

[35] Muller S, Saur R Greve B, Melms A, Hautzinger M, Fallgatter AJ, et al. Similar autobiographical memory impairment in long-term secondary progressive multiple sclerosis and Alzheimer's disease. Mult Scler 2013;19:225-32.

[36] Osterrieth PA. Le test de copie d'une figure complexe. Arch Psychol 1944;30:206-356.

[37] Paul R Blanco C, Hames K, Beatry VW. Autobiographical memory in multiple sclerosis. J Neuropsychol Int Soc 1997;3:246-51.

[38] Rao SM, Leo GJ, Bernardin L, Unverzagt F. Cognitive dysfunction in multiple sclerosis: I. Frequency, patterns, and prediction. Neurology 1991;41:685-91.

[39] Race E, Keane MM, Verfaellie M. Medial temporal lobe damage causes deficits in episodic memory and episodic future thinking not attributable to deficits in narrative construction. J Neurosci 2011;31:10262-9.

[40] Rasmussen AS, Habermas T. Factor structure of overall autobiographical memory usage: the directive, self and social functions revisited. Memory 2011;19:597-605.

[41] Raven JC Advanced progressive matrices, set 1. Manual. London: H. K. Lewis; 1958.

[42] Rey A. L'examen clinique en neuropsychologie. Paris: PUF; 1964

[43] Rey A. L'examen psychologique dans les cas d'encephalopathie traumatique. Arch Psychol 1941;28:286-340.

[44] Rogers JM, Panegyres PK. Cognitive impairment in multiple sclerosis: evidence-based analysis and recommendations. J Clin Neurosci 2007;14:919-27.

[45] Schacter DL, Addis DR Hassabis D, Martin VC, Spreng NR Spzunar KK The future of memory: remembering, imaging, and the brain. Neuron 2012;76:677-94

[46] Shallice T. Specific impairments of planning. Phil Trans R Soc Lond Biol 1982;298:199-209. 
Published in: Journal of the Neurological Sciences (2014), vol. 345, pp. 68-74.

Status: Postprint (Author's version)

[47] Shallice T, Evans ME. The involvement of the frontal lobes in cognitive estimation. Cortex 1978;14:294-303.

[48] Strauss E, Sherman EMS, Spreen O. A compendium of neuropsychological tests: administration, norms, and commentary. 3rd ed. NY: Oxford University Press; 2006.

[49] Stroop JR Studies of interference in serial verbal reactions. J Exp Psychol 1935;18:643-62.

[50] Suddendorf T, Corballis MC. Mental time travel and the evolution of the human mind. Genet Soc Gen Psychol Monogr 1997;123:13367.

[51] Suddendorf T, Corballis MC. The evolution of foresight: what is mental time travel and is it unique to humans? Behav Brain Sci 2007:30:299-313.

[52] Svoboda E, Hynes CA, Campbell AF, Dade LA Moscovitch M, Levine B. The frontal lobes and autobiographical memory: differential effects of dorsolateral and ventrolateral prefrontal damage. J Int Neuropsychol Soc 2002;8:275.

[53] Svoboda E, McKinnon MC, Levine B. The functional neuroanatomy of autobiographical memory: a meta-analysis. Neuropsychologia 2006;44:2189-208.

[54] Szpunar KK. Neural substrates of envisioning the future. Proc Natl Acad Sci 2007;104:642-7.

[55] Szpunar KK. Episodic future thought: an emerging concept. Perspect Psychol Sci 2010;5:142-62.

[56] Szpunar KK, Addis DR McLelland VC, Schacter DL. Memories of the future: new insights into the adaptive value of episodic memory. Front Behav Neurosci 2013;7:47.

[57] Trapp BD, Nave KM. Multiple sclerosis: an immune or a neurodegenerative disorder? Annu Rev Neurosci 2008;31:247-69.

[58] Tulving E. Memory and consciousness. Can Psychol 1985;26:1-12.

[59] Tulving E, Schacter DL, McLachlan DR Moscovitch M. Priming of semantic autobiographical knowledge: a case study of retrograde amnesia Brain Cogn 1988;8:3-20.

[60] Warrington E, James M. The Visual Object and Space Perception Battery (VOSP). Suffolk UK Thames Valley Test Company; 1991.

[61 ] Wechsler D. Manual for the Wechsler Adult Intelligence Scale — third edition (WAIS-II). San Antonio, TX: The Psychological Corporation; 1997.

[62] Williams JMG, Ellis NC, Tyers C, Healy H, Rose G, MacLeod AK The specificity of autobiographical memory and imaginability of the future. Mem Cogn 1996;24:116-25. 